\title{
Las redes académicas de Albrecht von Haller y la Sociedad Económica: un análisis de redes a varios niveles
}

\author{
Martin Stuber \\ Instituto de Historia, Universidad de Berna, Suiza ${ }^{1}$ \\ Lothar Krempel \\ Instituto Max Planck para el estudio de las sociedades, Colonia,
}

Alemania $^{2}$

\begin{abstract}
Resumen
En este artículo se ofrece un análisis de redes a varios niveles a través del ejemplo de la transferencia de plantas cultivadas dentro del contexto de las redes de correspondencia de Albrecht von Haller y la Sociedad Económica. Por medio de un procedimiento a varios niveles se analizan, una a una, la dinámica cronológica, la estructura social, la distribución espacial y la creación funcional de redes. Estos cuatro niveles de análisis de redes no están en conflicto unos con otros, sino que se complementan mutuamente. El objetivo consiste en comprender mejor cómo contribuyeron dichas redes a la transferencia internacional de conocimientos en el siglo XVIII.
\end{abstract}

Palabras clave: República de las Letras, Ilustración Económica, Transferencia de plantas útiles.

\begin{abstract}
Here, by the example of the transfer of cultivated plants in the context of the correspondence networks of Albrecht von Haller and the Economic Society, a multilevel network analysis is suggested. By a multi-level procedure, the chronological dynamics, the social structure, the spatial distribution and the functional networking are analyzed one after the other. These four levels of network analysis do not compete with each other but are mutually supporting. This aims at a deeper understanding of how these networks contributed to an international transfer of knowledge in the 18th century.

Keywords: Republic of Letters, Economic Enlightenment, Transfer of useful plants.
\end{abstract}

Este artículo versa sobre la red personal del erudito suizo Albrecht von Haller (1708-1777) y la red institucional de la Sociedad Económica (Oekonomische

\footnotetext{
El presente artículo se presentó en la conferencia internacional «Análisis de redes e historia: enfoques, herramientas y problemas», celebrada en Lausana entre el 22 y el 27 de febrero de 2010 . Otros artículos de dicha conferencia se han publicado en REDES - Revista hispana para el análisis de redes sociales, vol. 21, 7, diciembre de 2011. Los autores expresan su agradecimiento a Sandro Guzzi-Heeb (Lausanne), Claire Lemercier (Paris), Michel Bertrand (Toulouse) así como a los revisores anónimos por sus críticos y útiles comentarios, así como a Luis Sarabia (Madrid) por la versión final en español de este artículo.
}

1 Dirección de contacto: martin.stuber@hist.unibe.ch.

2 Dirección de contacto: $1 \mathrm{k} @$ mpifg.de. 
Gesellschaft), fundada en Berna en 1759. Por un lado, estas dos redes sirven en cada caso como bases empíricas independientes para el análisis de redes a varios niveles aquí propuesto. Por otro, se hará asimismo hincapié en las interacciones entre ambas redes, que, a primera vista, provienen de ámbitos distintos. La de Albrecht von Haller se debe encuadrar en la República de las Letras. Únicamente aquellas personas que hablaran latín, hubieran estudiado en la universidad y/o tuvieran una publicación académica editada podían pertenecer a ese sistema de comunicación del ámbito académico de principios de la Edad Moderna ${ }^{3}$. La Sociedad Económica, por su parte, se enmarca dentro de una Ilustración Económica con una base mucho más amplia que comenzó a abarcar extensas partes de Europa en la segunda mitad del siglo XVIII ${ }^{4}$. Tal y como se verá posteriormente, las conexiones y las coincidencias entre la República de las Letras y la Ilustración Económica demostraron ser mucho más amplias de lo que se pensaba ${ }^{5}$.

A continuación empezaremos con una breve presentación de los actores principales, un comentario sobre las fuentes históricas en las que se basa este estudio y una descripción de los datos y las bases de datos que sustentan nuestro análisis (1). Posteriormente se esbozarán dos ejemplos de transferencia cultural en red (2). Sobre esta base, el siguiente paso consistirá en abstraer lo anterior para trazar las distintas representaciones de redes, que deberían proporcionar las líneas generales del análisis de redes a varios niveles aquí propuesto. Con ello se pretende dejar patente que las distintas dimensiones de la dinámica cronológica, de la estructura social, de la extensión espacial y de la creación de redes son mutuamente ilustrativas, complementarias y extensivas (3).

\section{Principales actores y datos}

La primera red describe la correspondencia de Albrecht von Haller (fig. 1$)^{6}$. Después de estudiar en Tubinga y en Leiden empezó a ejercer la medicina en su ciudad natal, Berna. En 1736, Haller fue nombrado profesor de Anatomía, Botánica y Cirugía en la recién fundada Universidad de Gotinga. Además de la botánica, la fisiología pasó a ser su principal actividad de investigación. Al conceptualizar la fisiología como «anatomía animada», algo totalmente novedoso, se convirtió en una autoridad científica a nivel internacional. En 1753, Haller volvió a su país natal

\footnotetext{
Schneider 2005; Bosse 1997; Bots y Wacquet 1997.

Stapelbroek y Marjanen 2012; Popplow 2010; Lowood 1991.

Véase también Brockliss 2002.

Steinke et al. 2008.
} 
para trabajar de director en las minas de sal y, más tarde, en la Administración como miembro del Comité Económico y del Consejo Médico. Gracias a sus múltiples funciones como empleado público ilustrado, autor de tratados fundamentales (sobre plantas forrajeras, cereales y la peste bovina) y presidente de la Sociedad Económica de Berna, Haller fue una de las figuras clave de la Ilustración Económica en Berna.

Figura 1. Albrecht von Haller (1708-1777), 1760 aprox. - Burgerbibliothek de Berna

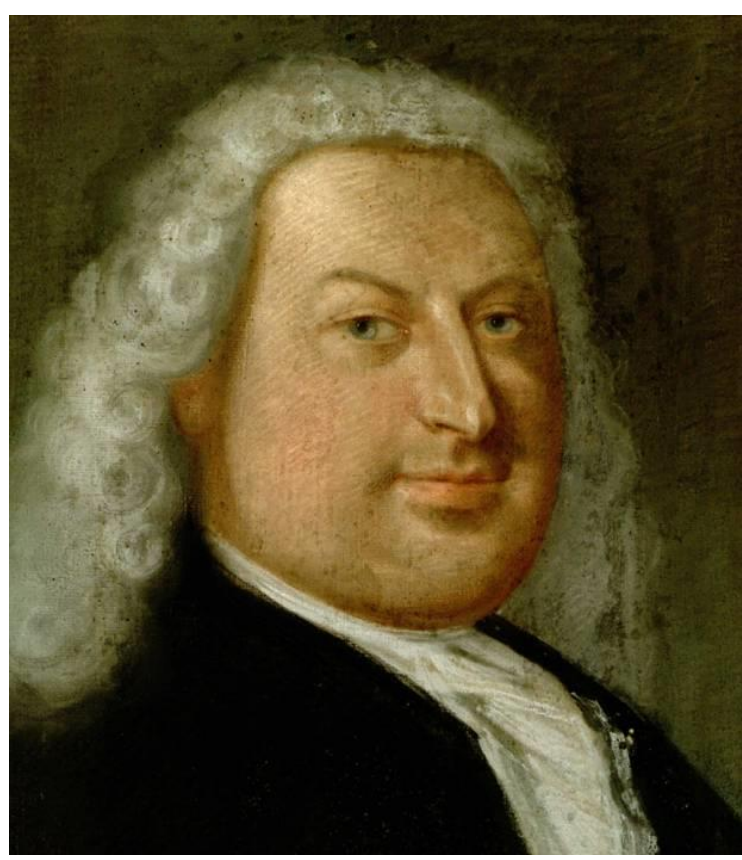

La Sociedad Económica de Berna (fig. 2) conforma la segunda red que se va a analizar7. Después de sus predecesoras en Edimburgo (1723), Dublín (1731), Florencia (1753), Londres (1754) y Rennes (1757), la fundación de la Sociedad Económica en 1759 vino acompañada de una auténtica oleada de nuevas sociedades de este tipo, que empezaron a abarcar extensas partes de Europa a partir de principios de los años 1760. Sus coetáneos ya consideraban la Sociedad Económica de Berna como una de las instituciones más importantes de la Ilustración Económica. Este movimiento de reforma agroeconómica a escala europea tenía como objetivo generar conocimientos útiles en torno a las reformas económicas y sociales. Los recursos más importantes son nuevas formas de comunicación en red que hacen que el conocimiento mundial y la experiencia local estén más estrechamente relacionadas ${ }^{8}$.

Stuber et al. 2009.

8 Holenstein, Stuber y Gerber-Visser 2007. 
Figura 2. Medalla honorífica de la Sociedad Económica, 1763. - Museo Histórico de Berna.

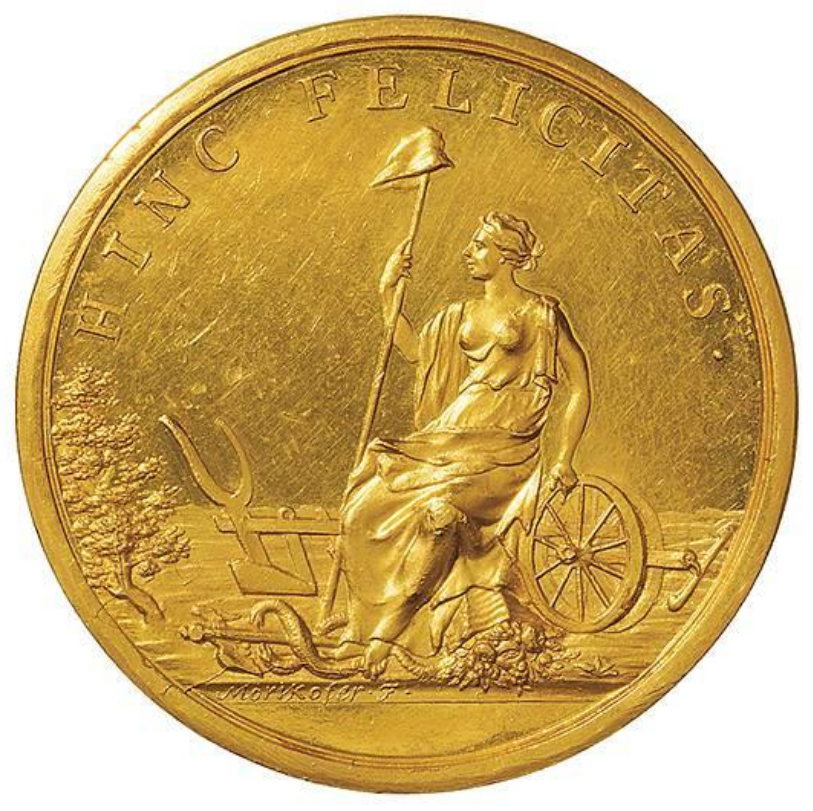

Nuestro análisis está basado empíricamente en dos proyectos de investigación de varios años de duración sobre Albrecht von Haller y la Sociedad Económica ${ }^{9}$. Los extensos datos obtenidos en ambos proyectos de investigación se han normalizado, homogeneizado e integrado en una base de datos combinada ${ }^{10}$.

Si bien el típico estudio de correspondencias históricas documenta patrones de comunicación de un único actor específico, esa mera transcripción egocéntrica proporciona únicamente una visión limitada del marco social. Las actividades de todos los corresponsales en las que no interviene el actor elegido pasan desapercibidas. Esto mismo es aplicable a la interacción entre dos redes de correspondencia de ese tipo. No obstante, estas limitaciones se pueden sortear documentando las actividades desde diversos puntos de referencia, con lo que se consigue una perspectiva multipolar de las redes.

Incrementar el rango de estudio de un enfoque egocéntrico a una perspectiva multipolar de las redes requiere mucha más mano de obra que la perspectiva

9 Proyecto de investigación Albrecht von Haller und die Gelehrtenrepublik (<www.haller.unibe.ch>, Instituto de Historia de la Medicina, Universidad de Berna, dirigido por Urs Boschung): 1991-2003; proyecto de investigación Nützliche Wissenschaft, Naturaneignung und Politik. Die Oekonomische Gesellschaft Bern im europäischen Kontext, 1750-1850 (<www.oeg.hist.unibe.ch>, Instituto de Historia, Universidad de Viena, dirigido por André Holenstein y Christian Pfister): 2004-2011. Ambos fueron financiados por la Swiss National Science Foundation.

10 Forschungsdatenbank zu Albrecht von Haller und zur Oekonomischen Gesellschaft Bern (ubicación: Instituto de Historia de la Medicina, Instituto de Historia, Burgerbibliothek de Berna); cf. Flückiger y Stuber 2009; Steinke 2003. 
individual. La integración de distintas bases de datos es una forma relativamente rentable de conseguir una visión más multipolar de las redes: almacenar la información de las redes $\mathrm{A}$ y $\mathrm{B}$ y los atributos de sus miembros y enriquecerlas con afiliaciones adicionales permite descubrir ambas redes dentro de su entorno común y mejora nuestra forma de comprender su interacción.

\section{Ejemplos de transferencia en red de plantas útiles}

Para ilustrar las ventajas de esta perspectiva multipolar ofrecemos dos ejemplos que identifican muchas coincidencias e interacciones en las actividades de la red personal de Albrecht von Haller y la red institucional de la Sociedad Económica.

\section{Transferencia de plantas del género Rubia}

En 1764, el fundador de la Sociedad Económica, Johann Rudolf Tschiffeli, leyó información acerca de una nueva técnica para el procesado de plantas del género Rubia en unos artículos de la Sociedad de Agricultura de Ruan ${ }^{11}$. Puede que a Tschiffeli le llegara dicho artículo a través de Albrecht von Haller, puesto que la revista estaba incluida en el catálogo de la biblioteca de Haller ${ }^{12}$. Las raíces de plantas del género Rubia -una materia prima para la producción del color rojotenían una gran importancia comercial. Tschiffeli escribió a Louis-Alexandre Dambourney, un comerciante de Ruan y autor del artículo, y le pidió consejo y unas cuantas semillas ${ }^{13}$. Tschiffeli recibió ambas cosas, leyó dicha carta en una reunión de la Sociedad Económica y trató de cultivar las semillas ${ }^{14}$. Poco después, Dambourney fue nombrado miembro honorífico de la Sociedad Económica ${ }^{15}$. Por su parte, Tschiffeli siguió uno de los consejos de Dambourney, y buscó variedades autóctonas de Rubia. Escribió a Albrecht von Haller, cuyo estudiante de botánica Johann Jakob Dick le aseguró que algunas de las plantas que crecían de forma natural en la zona de Wallis eran del género Rubia ${ }^{16}$. Al final, Tschiffeli recibió tallos y semillas de variedades autóctonas de Rubia por parte de Haller, de un corresponsal de Haller llamado Abraham-Louis Decoppet -un pastor de Aigle- y del

11 Según Stuber 2008a, pp. 252-263.

12 Dambourney 1763; Monti 1883-1994, núm. 7412.

13 Tschiffeli 1765.

14 Abhandlungen und Beobachtungen durch die Oekonomische Gesellschaft zu Bern gesammelt, 1765, 1, p. IX-XXXV (VS 1764-02-23; VS 1764-05-05).

15 Abhandlungen und Beobachtungen durch die Oekonomische Gesellschaft zu Bern gesammelt, 1765, 1 , p. IX-XXXV (VS 1764-03-10).

16 Carta de Tschiffeli a Haller, 28 de enero de 1764 (Burgerbibliothek de Berna). 
estudiante de Haller llamado Dick, de Spiez, que ya ha sido mencionado anteriormente ${ }^{17}$. Posteriormente, todos esos tallos de plantas silvestres del género Rubia fueron presentados en la reunión de la Sociedad Económica celebrada en junio de $1765^{18}$.

Sobre la base de estos múltiples procesos de transferencia, Tschiffeli publicó un documento muy completo sobre el género Rubia en la revista de la Sociedad Económica ${ }^{19}$. Dos años más tarde apareció un fragmento de dicha publicación en la revista de Ruan, donde Tschiffeli había iniciado su labor tres años antes ${ }^{20}$. Esta exitosa transferencia se desarrolló al mismo tiempo por las vías de comunicación institucionales de la Sociedad Económica -reuniones, cartas y publicaciones- y las redes de correspondencia privada de Haller y Tschiffeli. El resultado fue un nuevo bagaje revestido claramente de una importancia supralocal, y no tardó mucho tiempo en aplicarse con éxito en el ámbito comercial. En 1765, Tschiffeli pudo anunciar con orgullo que ya había 12 familias que estaban ganándose la vida con sus cultivos de plantas del género Rubia en Kleehof (Kirchberg). En 1767 le escribió a un amigo que había vendido más de 300 centenas solo en ese año; y se prometieron 1000 centenas a los distintos manufactureros para el año siguiente ${ }^{21}$.

\section{Transferencia de zanahoria}

En julio de 1765, Peter Collinson, un empresario londinense y uno de los actores clave en la transferencia internacional de plantas de su época, le envió a Albrecht von Haller, con el que llevaba mucho tiempo carteándose, un artículo sobre el cultivo de zanahorias y su uso como pienso animal ${ }^{22}$. El autor del artículo era Robert Billing, un agricultor de Norfolk. Ese mismo año lo publicó a petición de la Sociedad para el Fomento de las Artes, la Manufactura y el Comercio de Londres ${ }^{23}$. En agosto de 1765 se leyó una carta ante el comité de la Sociedad Económica que llevaba anexo ese mismo artículo ${ }^{24}$. En marzo de 1766, el comité decidió traducir dicho artículo del inglés al francés y al alemán y publicarlo en su revista, donde

Tschiffeli 1765, p. 154.

18 Abhandlungen und Beobachtungen durch die Oekonomische Gesellschaft zu Bern gesammelt, 1766, 1, V-XXVI (VS 1765-06-17).

19 Tschiffeli 1765.

20 Dambourney 1765.

21 Tschiffeli 1765.

22 Según Stuber 2008a, pp. 244-245; carta de Collinson a Haller, 18 de julio de 1765 (Burgerbibliothek de Berna).

23 Billing 1765; véase Monti 1983-1994, núm. 900.

24 Carta de Templeman a la Oekonomische Gesellschaft, 24 de agosto de 1765v (Abhandlungen und Beobachtungen durch die Oekonomische Gesellschaft zu Bern gesammelt 1766, 1, p. XIX-XX). 
apareció respectivamente como «Relation sur la culture des carottes jaunes» y «Nachricht von dem Anbaue der Möhren» en $1767^{25}$. A finales de ese mismo año, el reverendo Johann Jakob Gerwer de Vinelz envió una carta a la Sociedad Económica -que se publicó poco después- explicando cómo había conseguido cultivar zanahorias, «para lo cual el artículo del señor Billing ha sido fuente no solo de motivación, sino también de orientación» ${ }^{26}$. François-Joseph-Antoine de Hell, alguacil de Alta Alsacia y miembro honorífico de la Sociedad Económica, era un ávido lector de ambas publicaciones. En su carta, fechada en diciembre de 1768 , se quejaba a la Sociedad Económica de que sus zanahorias no habían crecido como se describía en Abhandlungen und Beobachtungen. Según sus sospechas, sus semillas eran de una variedad más pobre que las que utilizaban Billing y Gerwer, y le pidió semillas de buena calidad a la sociedad bernesa a cambio de dinero ${ }^{27}$. Al parecer, dicha solicitud fue remitida directamente al sacerdote Gerwer de Vinelz, ya que en enero de 1769 ya le había respondido. Ordenó a su feligresía que obtuvieran cantidades suficientes de semillas de zanahoria, que, posteriormente, reenviaría a la atención del señor Hell ${ }^{28}$. En marzo de ese mismo año, Gerwer pudo, por tanto, enviar las semillas necesarias al secretario Thormann. En la carta que las acompañaba escribió: «c'est tout que j'ai pu ramasser avec toutes les peines que je me suis donné ${ }^{29}$ [es todo lo que he podido reunir con todos los esfuerzos que he realizado]. Y, a su vez, fueron reenviadas puntualmente. Ya en abril de 1769, Hell tuvo la oportunidad de darle las gracias a la sociedad bernesa por las semillas de zanahoria, que habían llegado justo a tiempo para la siembra ${ }^{30}$.

\section{Transferencia cultural en red}

Los dos ejemplos expuestos anteriormente se pueden interpretar dentro del contexto del paradigma de investigación de los procesos de transferencia cultural. $\mathrm{Si}$ bien el estudio de dichos procesos ha estado durante mucho tiempo conceptualizado como el intercambio entre al menos dos culturas nacionales espaciales homogéneas, los académicos están promoviendo actualmente una mayor concienciación con respecto a la variedad y al carácter reticular de estas

25 Abhandlungen und Beobachtungen durch die Oekonomische Gesellschaft zu Bern gesammelt 1767, 1, p. XIII-XXIX (VS 1766-03-15); Billing 1767.

26 Carta de Gerwer a la Oekonomische Gesellschaft, 31 de diciembre de 1767 (Burgerbibliothek de Berna): «Wozu Hrn. Billings Abhandlung nicht nur den Anlass, sondern auch die nöthige Anleitung gegeben.»; Gerwer 1768.

27 Carta de Hell a la Oekonomische Gesellschaft, 12 de diciembre de 1768 (Burgerbibliothek de Berna).

28 Carta de Gerwer a la Oekonomische Gesellschaft, 26 de enero de 1769 (Burgerbibliothek de Berna).

29 Carta de Gerwer a la Oekonomische Gesellschaft, 15 de marzo de 1769 (Burgerbibliothek de Berna).

30 Carta de Hell a la Oekonomische Gesellschaft, 23 de abril de 1769 (Burgerbibliothek de Berna). 
interacciones $^{31}$. En ambos casos -tanto en el de las plantas del género Rubia como en el de la zanahoria- se observan las secuencias básicas de una transferencia cultural (selección, transferencia y recepción) en distintas partes de las redes. Las sociedades de Berna, Ruan y Londres, así como sus publicaciones, terminan siendo las plataformas que organizan, traducen, multiplican y sancionan las actividades de los actores particulares. Gracias a ambas bases de datos podemos identificar partes de una red que vincula las esferas de la República de las Letras con la Ilustración Económica.

\section{Representaciones de redes}

A continuación vamos a relacionar esos dos ejemplos concretos de transferencias de plantas con los niveles relativamente abstractos del análisis de redes ${ }^{32}$. Con nuestro enfoque de un análisis de redes a varios niveles proponemos estudiar simultáneamente la dinámica cronológica, la estructura social, la dimensión espacial y la interrelación de los ámbitos. Las primeras partes del análisis siguen la tradición de Daniel Roche, que consideraba una red de correspondencia como un organismo vivo: dicho organismo tiene un comienzo y un final cronológicos, posee su propia estructura social y espacial y es intercambiable por muchos otros organismos del mismo tipo ${ }^{33}$. Para analizar las interrelaciones hacemos uso de métodos más recientes de las ciencias sociales (concretamente, procedimientos de visualización de redes ${ }^{34}$, cuyo potencial ya hemos probado en otro estudio ${ }^{35}$ ).

\section{Dinámica cronológica}

La primera dimensión analítica está constituida por la dinámica temporal que exhiben ambas redes, lo que requiere un debate más a fondo sobre la exhaustividad de los datos disponibles. Esto lo consideramos algo especialmente importante, puesto que al análisis histórico de redes se le acusa con frecuencia de hacer caso omiso tanto de la dimensión cronológica como de las pruebas históricas.

31 Reichardt 2003, p. 28; véase Lüsebrink 2001, pp. 213-226.

32 En el caso de Albrecht von Haller, el análisis puede sustentarse en publicaciones anteriores relativas a la época, la estructura social y el espacio: Stuber, Hächler y Steinke 2005. Las conexiones entre los actores, tal y como se exponen en los ejemplos utilizados inicialmente, se pueden considerar cadenas (véase Grossetti, Barthe y Chauvac 2011). No obstante, desde el punto de vista adoptado aquí, se pueden identificar como actores en red que contribuyen de forma tópica con las actividades en red en un momento dado.

33 Roche 1971, pp. 151-172.

34 Krempel 2006.

35 Stuber, Hächler, Krempel y Ruisinger 2008. 
En total, Haller intercambió 16981 cartas con 1138 corresponsales hombres, 50 corresponsales mujeres y 11 instituciones. Si bien Haller recibió de parte de sus corresponsales 13.237 cartas, no hay nada más que 3.724 enviadas, principalmente debido a la disponibilidad de los registros; en general, cabe suponer que Haller hubiera respondido a todas las cartas. Por consiguiente, sólo las cartas recibidas de parte de sus corresponsales se pueden utilizar para llevar a cabo un análisis estadístico. En lo que a ellas respecta podríamos asumir que contamos con pruebas suficientes, pero no con la totalidad de ellas. Las notas de muestreo de su diario $(1739,1745$ y 1746), en las que Haller realizó un listado de todas las cartas que recibía, nos permiten asumir que prácticamente dos tercios de todas estas cartas sobrevivieron ${ }^{36}$. Durante sus primeros años, cuando Haller trató deliberadamente de establecer una red de correspondencia científica viajando y ofreciendo servicios ${ }^{37}$, el número de cartas incrementó hasta rondar las 60 cartas recibidas al año. Tras su nombramiento en Gotinga, esa cifra aumentó a 200 cartas recibidas. El punto máximo se registró durante los años 1752-53, con más de 500 cartas, en relación con la polémica a escala europea en torno a su teoría de la irritabilidad. Tras su vuelta a Berna, el número de cartas recibidas se estabilizó en una cifra elevada: entre 250 y 300 cartas al año ${ }^{38}$.

La red de correspondencia de la Sociedad Económica incluye muchas menos cartas. Para el periodo comprendido entre 1759 y 1800 hay 1044 cartas escritas a la Sociedad Económica disponibles en la Burgerbibliothek de Berna. A partir de las actas de las reuniones hemos logrado reconstruir 792 más (fig. 3), de las cuales no hay ningún documento en los archivos. Cabe, por tanto, esperar que el desarrollo temporal reconstruido a partir de los documentos disponibles refleje con exactitud las actividades históricas, y no debería sorprendernos que los años de intensa actividad social de la década de 1760 y principios de la de 1770 sean asimismo un período con un gran flujo de correspondencia ${ }^{39}$.

\footnotetext{
36 Boschung 2002; Stuber (en la versión impresa).

Stuber 2005.

Stuber, Hächler y Steinke 2005, pp. 65-84.

Stuber 2008b, p. 126; Salzmann 2009.
} 
Figura 3. Red de correspondencia de la Sociedad Económica: desarrollo temporal (Stuber 2008b, p. 127).

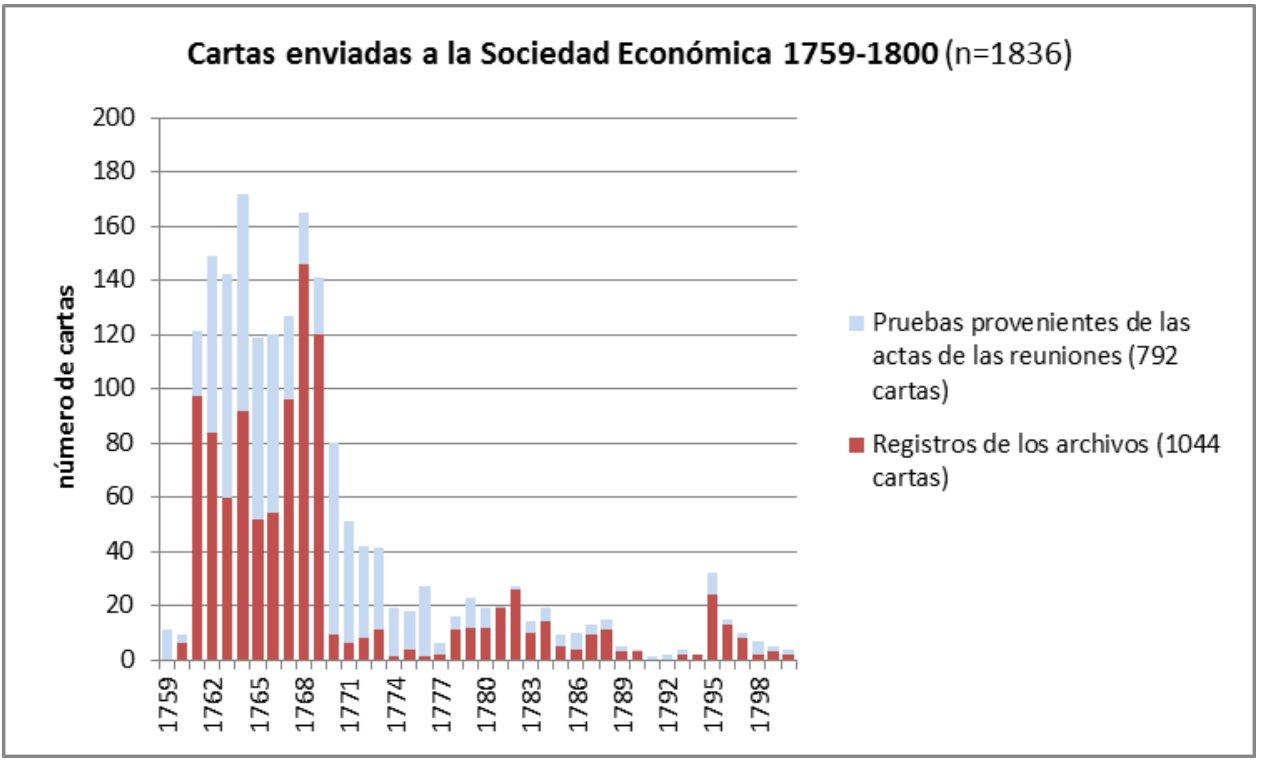

De este periodo de intensa actividad en redes por parte de la Sociedad Económica datan asimismo los dos ejemplos citados inicialmente de la transferencia de las plantas del género Rubia (1764-67) y de la zanahoria (1765-69). Para el periodo comprendido entre 1759 y 1782 es posible reconstruir un total de 209 actividades relacionadas con la transferencia de semillas, raíces y vástagos, en la mayoría de los casos a partir de cartas escritas directamente a la Sociedad Económica (143 casos) y otras, de sus tratados (21), de las actas de las reuniones (21), así como de cartas a Haller $(28)^{40}$. En las décadas de 1760 y 1770 también se elaboraron inventarios sistemáticos de plantas útiles en el entorno de la Sociedad Económica y con Albrecht von Haller como actor clave-, 11 inventarios sistemáticos de plantas que relacionaban cerca de 650 especies distintas con sus nombres locales o internacionales ${ }^{41}$. Tal y como queda ilustrado por estos ejemplos, la transferencia cultural en red se basaba en un sistema translocal de referencias de este tipo.

\section{Estructura social}

En una segunda fase analizamos la composición de las redes de correspondencia con ayuda de atributos adicionales. Al hacerlo, el ejemplo de la red de correspondencia de Haller sirve como característica absoluta (posición profesional);

40 Stuber 2008a, p. 239.

41 Stuber y Lienhard 2007. 
mientras que, por otro lado, la red de la Sociedad Económica sirve como característica relacional (calidad de miembro) ${ }^{42}$.

La figura 4 proporciona información acerca de las profesiones de los 1188 corresponsales, sopesadas con respecto al total de 13226 que escribieron a Albrecht von Haller. La categoría profesional más frecuente de los corresponsales es la de médico, con un $31 \%$. Para evaluar este resultado se necesitan redes adicionales para las que existen estudios parecidos como, por ejemplo, las redes de Esprit-Claude-François Calvet, Gotthold Ephraim Lessing, Jean-Jacques Rousseau y Christoph Jakob Trew $^{43}$. Entre ellas, la red de correspondencia entre el médico y editor Trew, de Núremberg es, desde una perspectiva social, la más interrelacionada, puesto que la comunicación médica predomina en ambas redes. El $72 \%$ de las cartas de Trew están escritas a personal médico, mientras que en el caso de las cartas de Haller esa cifra se sitúa en el $43 \%$. El $16 \%$ de las cartas de Haller están dirigidas a hombres de Estado, magistrados y responsables de la Administración, en contraste con tan solo un $8 \%$ de las de Trew. También se observa un elevado grado de comunicación con magistrados en la red de Calvet, un médico de Aviñón (14\%). No obstante, la parte más importante de sus cartas está dirigida a clérigos católicos (32\%), aparte de los médicos y los farmacéuticos, con los que intercambió un $30 \%$ de sus cartas. La estructura de la red de Rousseau es totalmente distinta: tan solo un $3 \%$ son médicos, mientras que los sacerdotes católicos y los pastores protestantes reciben un $5 \%$ de sus cartas cada uno. En su comunicación predominan la nobleza, los oficiales militares y los funcionarios de justicia (36\%). Otro tipo de red es la de Lessing, en la que observamos que un $14 \%$ de los corresponsales provienen de la nobleza y tan solo un $2 \%$ son expertos en ciencias naturales. El grupo más importante de los corresponsales de Lessing está constituido por eruditos ( $40 \%$ ) como profesores de universidad, educadores y clérigos, seguidos de escritores, funcionarios del Estado (13\%) y editores e impresores $(10 \%)^{44}$.

42 Distinción según Jansen 2006, pp. 51-54; véanse también los análisis relacionados de René Sigrist de las redes de botánicos del siglo XVIII, donde se hace hincapié en las características absolutas de los actores de las redes: Sigrist en la versión impresa; y, por otro lado, sus características regionales: Sigrist y Widmer 2011.

43 Brockliss 2002; Roche 1971; Bödeker 1987; Steinke 2000.

44 Stuber, Hächler y Steinke 2005, pp. 90-92. 


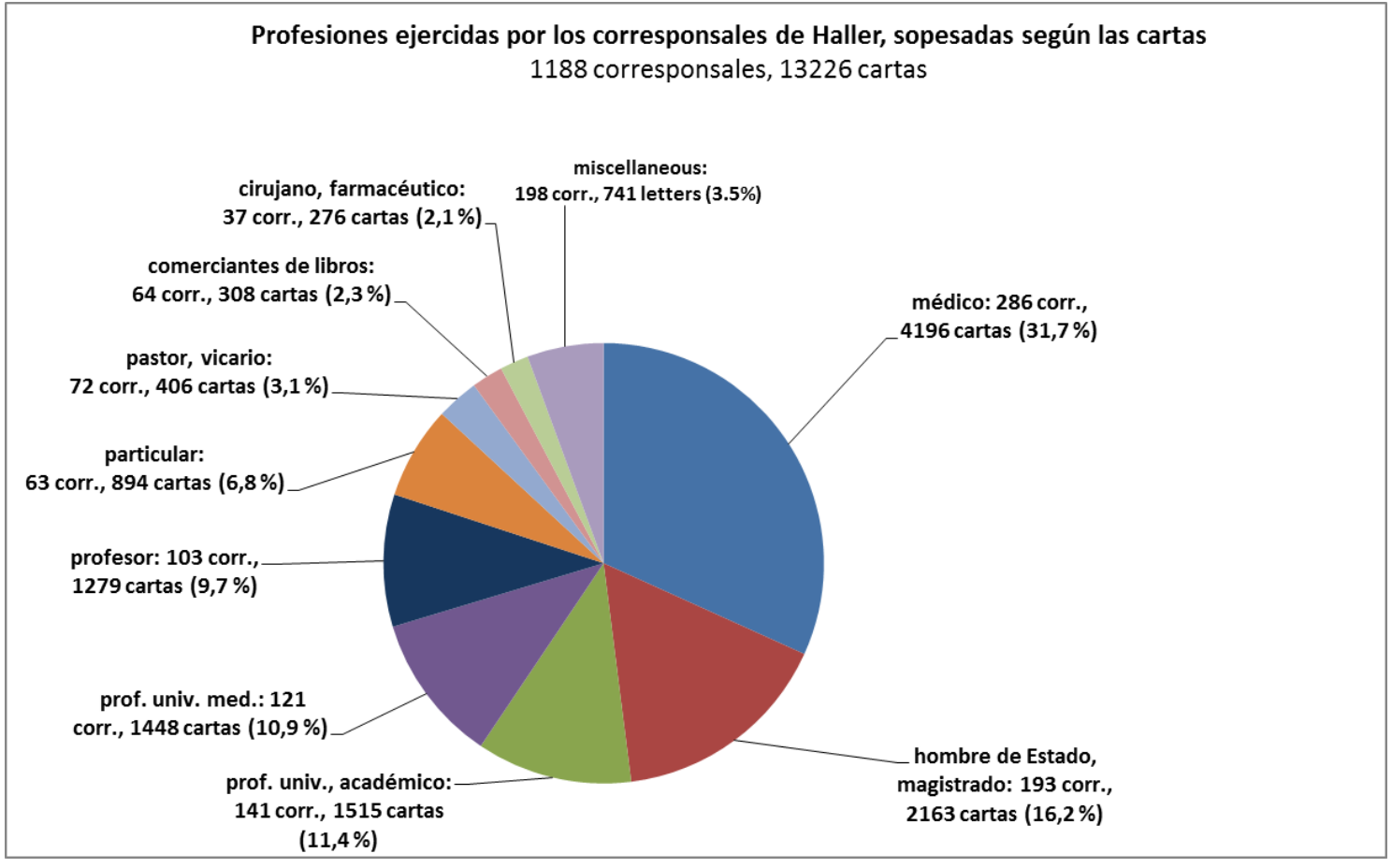

Aunque puede que la base de nuestros cinco ejemplos sea demasiado pequeña como para obtener una tipología estricta, los ejemplos sí que facilitan una orientación aproximada: Trew, Calvet y Haller forman parte principalmente de la República de las Letras, mientras que a Rousseau y a Lessing se les puede considerar representantes de un público culto e ilustrado más amplio ${ }^{45}$. No obstante, un elemento común a todas las redes es la elevada proporción de hombres de Estado, magistrados y responsables de la Administración, lo que indica una incrustación generalizada de la comunicación dentro de la estructura gubernamental. Esto queda ilustrado asimismo por los actores que contribuyeron con la transferencia de plantas útiles citada inicialmente dentro del contexto de las redes de Haller como elementos cercanos al Estado: los dos vicarios asalariados por el Estado Johann Jakob Dick y Abraham-Louis Decoppet, así como el responsable de la Administración Johann Rudolf Tschiffeli y el magistrado Albrecht von Haller.

Además de su estatus profesional, los organismos de los que eran miembros facilitan una mayor comprensión de las distintas funciones dentro de la red ${ }^{46}$. Un tercio de todas las cartas enviadas a la Sociedad Económica fueron escritas por miembros honoríficos, la mayoría de los cuales eran contactos internacionales. Otros corresponsales sin estatus institucional formal en la Sociedad Económica

45 Las fronteras eran bastante permeables: véase, por ejemplo, Bensaude-Vincent y Bernardi 2003.

46 Según Stuber 2008b, pp. 128-130. 
escribieron un $30 \%$ de las cartas, lo que revela las fronteras flexibles de esta red de comunicación. Queda por explicar el hecho de que casi el $20 \%$ de todas las cartas estuvieran escritas por miembros ordinarios, dado que supuestamente ellos podían expresar sus preocupaciones personalmente durante las reuniones de la sociedad. La explicación se halla en la forma en la que estaba organizada la Administración bernesa, que se regía por la movilidad cronológica del patriciado de la ciudad, de donde eran reclutados la mayoría de los miembros ordinarios. Tenían que gobernar durante un periodo de seis años en la zona rural de Berna, por lo que su participación se manifestaba principalmente en forma escrita.

Por último se ha de mencionar el $16 \%$ de las cartas provenientes de las filiales locales (Zweiggesellschaften). Fue el intercambio constante de información con estas ramas locales el que se difundió por todo el territorio bernés para cumplir el deseo de transferencia espacial del conocimiento. Muchos miembros de estas filiales eran vicarios que vivían en la zona rural de Berna. Entre ellos estaban Abraham-Louis Decoppet, Johann Jakob Dick y Jakob Gerwer, que también participaron en la transferencia de las plantas del género Rubia y las zanahorias. Asimismo, el resto de actores que aparecen en el contexto de los ejemplos de la transferencia de plantas útiles mencionados inicialmente podrían incluirse en estos grupos en calidad de miembros ordinarios de la Sociedad Económica, por un lado, como Albrecht von Haller, Johann Rudolf Tschiffeli y Victor Alex Thormann, y, por otro, en calidad de miembros honoríficos internacionales, como Louis-Alexandre Dambourney (Lyon), François-Joseph-Antoine de Hell (Alsacia) y Peter Templeman (Londres).

Por tanto, por su estructura social, en general los actores de la transferencia de plantas representan por un lado el fuerte arraigo de la Ilustración Económica en la política y en la Administración locales y, por otro, en la creación internacional de redes representada por los miembros honoríficos ${ }^{47}$.

\section{Distribución espacial}

Al igual que la de Albrecht von Haller, la red de la Sociedad Económica se extiende desde el Loira hasta el Óder, y ambas cuentan con una representación relativamente numerosa al sur de los Alpes, en las Islas Británicas y en Escandinavia (fig. 5). Ambas -la red de correspondencia de Albrecht von Haller y la

47 Véase Stuber 2012. 
de la Sociedad Económica- están revestidas de una dimensión europea ${ }^{48}$. Y eso es precisamente lo que las diferencia de otras redes de correspondencia. Un ejemplo del tipo no europeo es la red de correspondencia de Rousseau, que se limita básicamente a Francia y a la Suiza francófona y que únicamente cuenta con una representación marginal al sur de los Alpes, al este del Rin y en las Islas Británicas. Lo mismo se puede afirmar de la red de Christoph Jakob Trew, que no incluye ninguna ubicación al oeste del Rin.

Figura 5. Red de correspondencia de la Sociedad Económica (Stuber 2008b, pp. 127).

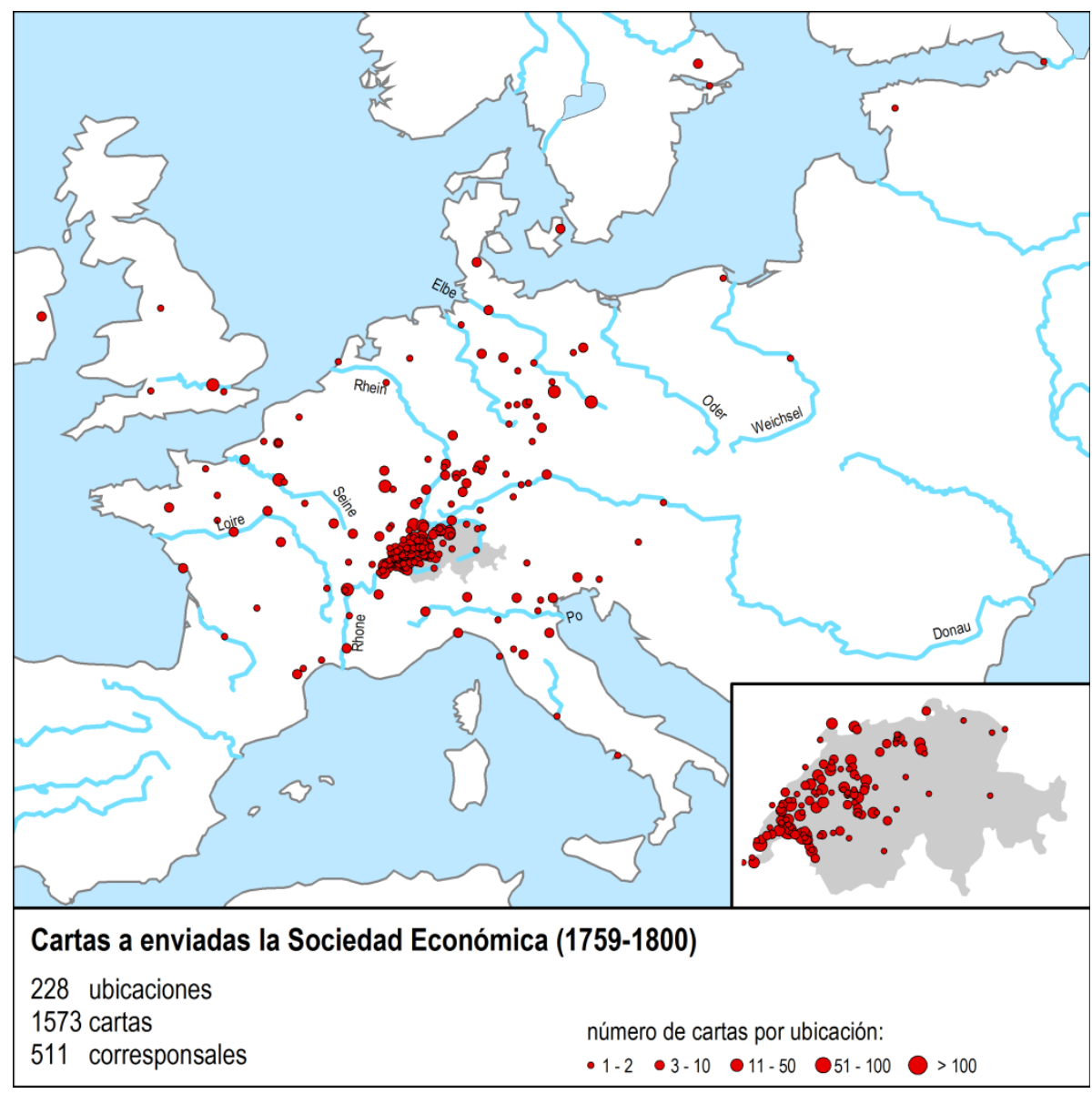

Asimismo, la transferencia de plantas, que es lo que ha puesto de relieve aquí, se basaba en esas redes a gran escala. Incluso aunque solo se consideren aquellas actividades en red relacionadas con las plantas, precisamente esa dimensión europea se les puede asignar tanto a la correspondencia botánica de Albrecht von Haller ${ }^{49}$ como a la transferencia de plantas útiles de la Sociedad Económica ${ }^{50}$. Y los ejemplos que hemos citado inicialmente también abarcan una región igual de extensa. La especie no autóctona de zanahorias se transfirió desde las Islas

48 Según Stuber, Hächler y Steinke 2005, pp. 31-40; Stuber 2008b, pp. 126-127.

49 Hächler 2008, pp. 201-218.

50 Stuber 2008b, pp. 240-241. 
Británicas (Norfolk) hasta Alsacia a través de Vinelz, y la nueva forma de cultivar y procesar las plantas del género Rubia viajó de Lyon a Kirchberg, cerca de Berna, y volvió a Lyon modificada.

\section{Interrelación}

Las investigaciones de Holger Zaunstöck ya destacaron la suma importancia de la creación de redes para la cultura de las comunicaciones del siglo XVIII y, concretamente, de la Ilustración ${ }^{51}$. Eso también dejó patente que no es conveniente limitarse a la red egocéntrica, que incluye únicamente la correspondencia recibida por la institución central. De hecho, el punto de partida debe ser una red multipolar que incorpore relaciones e interacciones con otras redes de correspondencia.

Tabla 1. Fechas de los corresponsales.

\begin{tabular}{|c|c|c|c|c|c|c|c|c|c|c|c|c|c|c|c|}
\hline & 1680 & 1690 & 1700 & 1710 & 1720 & 1730 & 1740 & 1750 & 1760 & 1770 & 1780 & 1790 & 1800 & 1810 & 1820 \\
\hline \multicolumn{16}{|l|}{ Heister } \\
\hline \multicolumn{16}{|l|}{ Trew } \\
\hline \multicolumn{16}{|l|}{ Linné } \\
\hline \multicolumn{16}{|l|}{ Haller } \\
\hline \multirow{2}{*}{\multicolumn{16}{|c|}{ Rousseau }} \\
\hline \multirow{2}{*}{\multicolumn{16}{|c|}{ Banks }} \\
\hline & & & & & & & & & & & & & & & \\
\hline Sociedad & & & & & & & & & & & & & & & \\
\hline Económica & & & & & & & & & & & & & & & \\
\hline
\end{tabular}

Aparte de la red de Albrecht von Haller y la de la Sociedad Económica, hemos incluido otras cinco redes de correspondencia en nuestra base de datos, las cuales están todas correctamente documentadas y comparten un enfoque botánico. Nos referimos a las redes de Joseph Banks, Lorenz Heister, Carl von Linné, JeanJacques Rosseau y Christoph Jacob Trew ${ }^{52}$ (Tab. 1). Además de las siete figuras principales, aparecen los corresponsales de todas las redes que intercambiaron cartas con al menos tres de los actores principales (fig. 6). Haller y Linné tienen la cifra más elevada de conexiones de ese tipo y, por tanto, aparecen definidos en la imagen como los dos círculos más grandes. La visualización está organizada espacialmente de acuerdo con el siguiente principio: los actores estrechamente vinculados entre sí están organizados cerca unos de otros, mientras que los actores que no están conectados o solo lo están por medio de largos caminos se

51 Zaunstöck y Meumann 2003; Zaunstöck 1999.

52 Según Stuber, Hächler, Krempel y Ruisinger. 
encuentran a cierta distancia entre sí. Un análisis más exhaustivo permite descubrir a los corresponsales de la interfaz de la República de las Letras y de la Ilustración Económica como, por ejemplo, Vautravers, que se carteaba con Rousseau, Banks, Linné, Haller y la Sociedad Económica; o el famoso agrobotánico Duhamel du Monceau, que se escribía con Linné, Haller y la Sociedad Económica; así como el magistrado y economista bernés Samuel Engel, que mantenía correspondencia con Haller, Linné y la Sociedad Económica. Aparte de Haller, de los actores de los ejemplos mencionados inicialmente tan solo se halla en la visualización Peter Collinson, que mantenía contactos indirectos con la Sociedad Económica a través de Haller y se carteaba con Linné y con Trew. No obstante, no debemos olvidarnos de que esta visualización se debe interpretar exclusivamente de forma positiva teniendo en cuenta la correspondencia escrita existente- y no negativa, es decir, teniendo en cuenta la correspondencia escrita perdida. En primer lugar, solo la correspondencia escrita de los siete actores clave está representada; en segundo lugar, se ha fijado un valor mínimo de tres relaciones de ese tipo; y, en tercer lugar, solo se puede visualizar aquella correspondencia escrita de la que existan pruebas históricas. Esta restricción minima tiene como consecuencia, por ejemplo, que Johann Jakob Dick, que aparecía en el ejemplo de las plantas del género Rubia, no esté presente en la visualización. De hecho, Dick estaba muy involucrado en las redes, como lo demuestra el hecho de que mantuviera correspondencia con al menos cinco de los corresponsales aquí mencionados (Michel Adanson, Petter Jonas Bergius, Johannes Gessner, Paul Dietrich Giseke y Johann Andreas Murray). Pero solo hay pruebas de dos correspondencias escritas con actores clave (Haller y la Sociedad Económica). No obstante, lo más probable es que también se carteara con Linné, puesto que en el herbario de este último se encuentran más de 30 pruebas de plantas suministradas por Dick. 
Figura 6. Visualización A: redes de correspondencia de botánicos del siglo XVIII (Stuber, Hächler, Krempel y Ruisinger 2008, pp. 352).

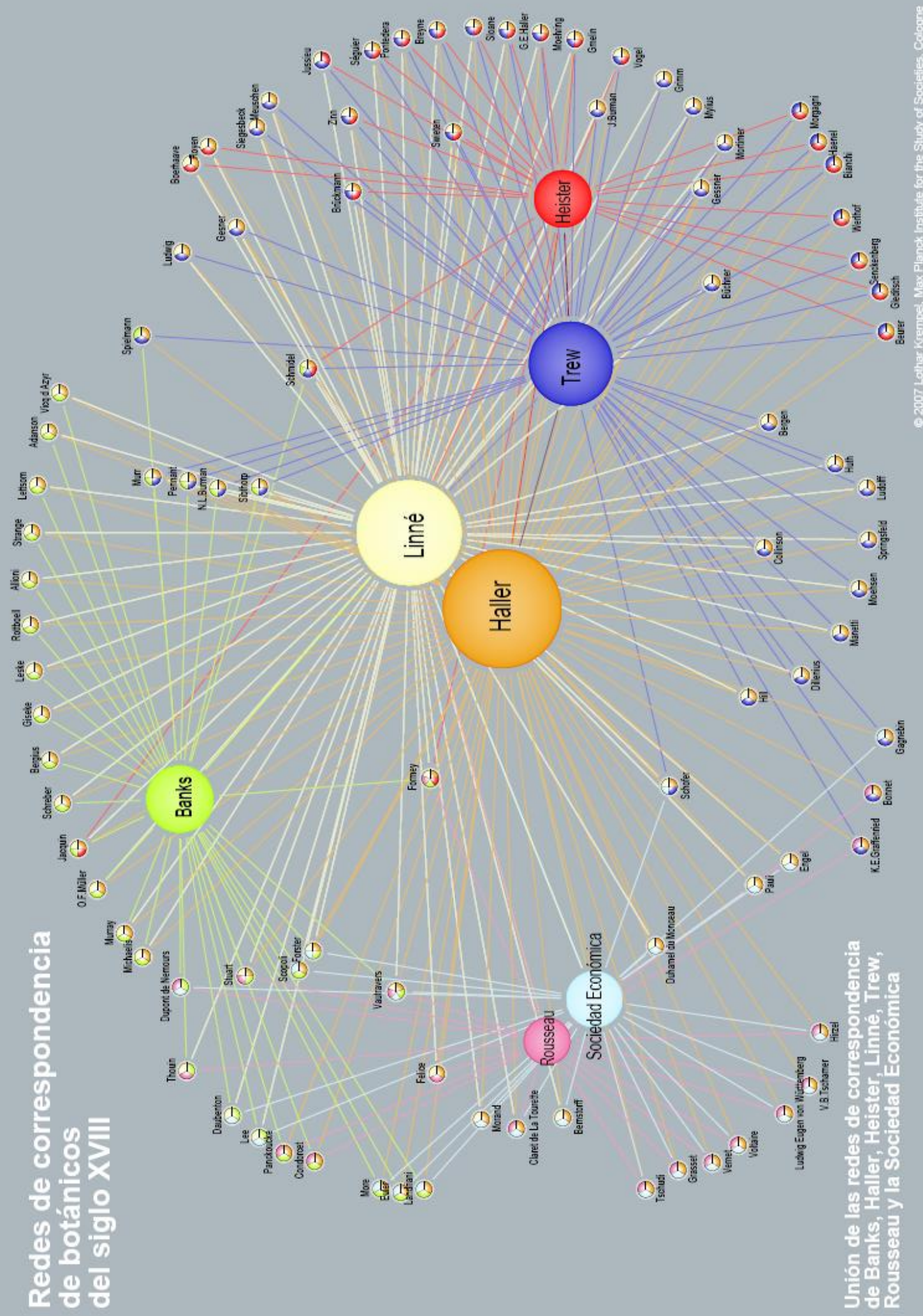


Figura 7. Visualización B: Movimiento reformista bernés, 1765.

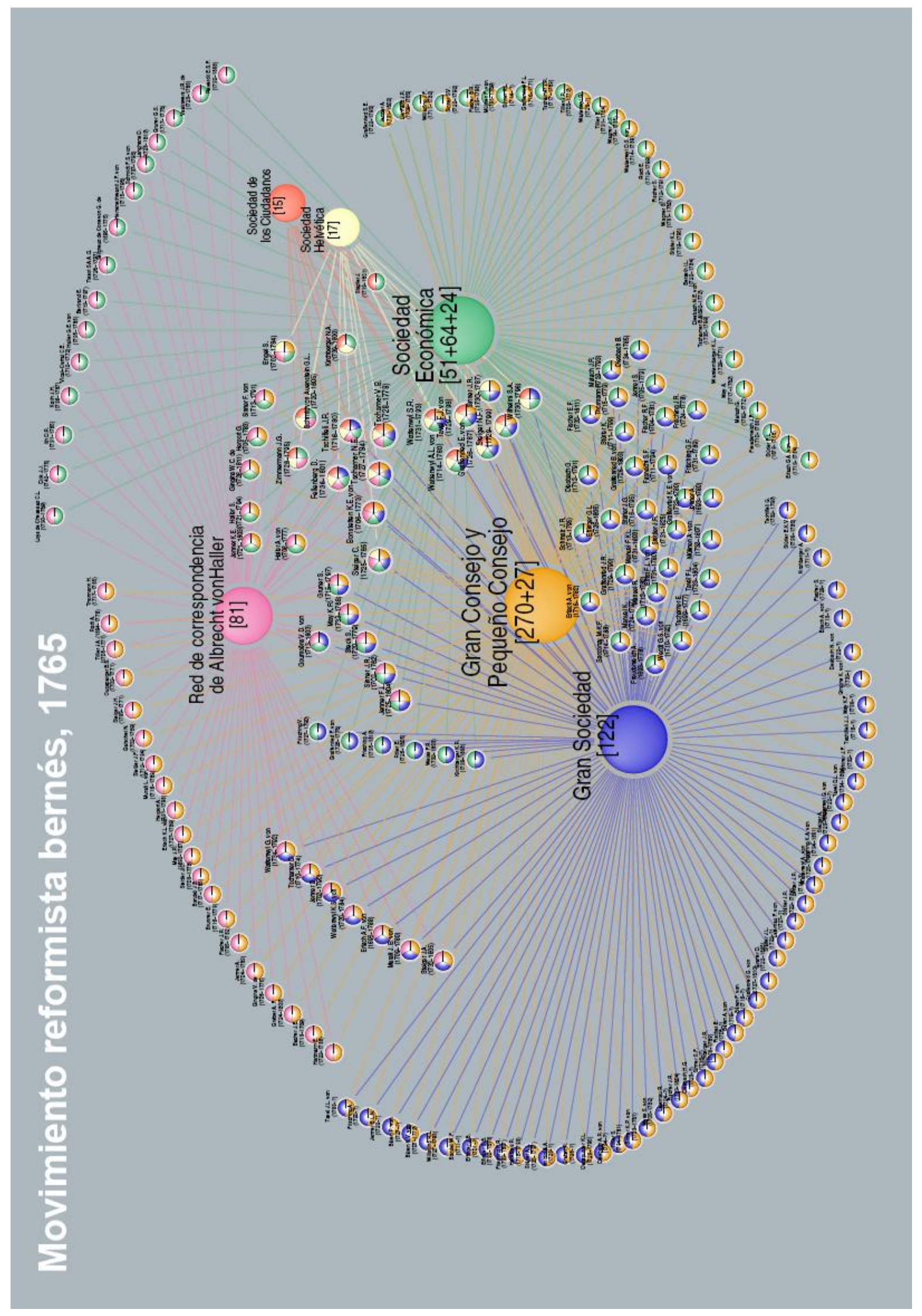


Si bien la primera visualización describe las relaciones directas entre los autores de las cartas, una segunda vía para potenciar la reconstrucción de datos históricos es un grafo bimodal que describe las relaciones entre dos conjuntos distintos de conceptos. Utilizamos dicho modelo para analizar con más detalle el movimiento reformista bernés. Aunque seguimos fijándonos en los autores de las cartas (primer conjunto), ahora utilizamos información adicional sobre las afiliaciones de los corresponsales con varias instituciones sociales (segundo conjunto) que son esenciales para comprender la sociedad bernesa del siglo XVIII. Estas instituciones están representadas mediante códigos de colores en la figura 7. El azul representa la Gran Sociedad (Grande Société) como centro social del patriciado ${ }^{53}$; el amarillo, el Gran Consejo y el Pequeño Consejo como el núcleo político de la República Bernesa; el verde, la Sociedad Económica como la institución reformista más poderosa; el blanco (a la derecha), la Sociedad Helvética (Helvetische Gesellschaft) como institución suiza de fuerzas reformistas a escala nacional ${ }^{54}$; el rojo, la radical Sociedad de los Ciudadanos (Société des Citoyens ${ }^{55}$ ); y el violeta, la red de correspondencia de Albrecht von Haller, restringida a sus corresponsales de Berna, Argovia y Vaud.

Si dos actores están vinculados exactamente al mismo grupo, pueden estar en contacto entre sí mediante dos pasos. Si esa es la única información que hay disponible de ellos, no se pueden distinguir a nivel relacional: son equivalentes. Los actores que son miembros de varias instituciones se sitúan en el centro de gravedad de todo aquello a lo que están conectados. Del mismo modo, dos organismos están situados cerca el uno del otro si la composición de sus actores coincide en gran medida; mientras que si ninguno o solo algunos de sus miembros coinciden, estarán a una distancia respectiva.

No obstante, debido a restricciones de espacio, del total de 392 actores solo hay ilustrados 188, los que cuentan al menos con dos relaciones. En la parte izquierda de la imagen se hallan todos los actores que no tienen ningún vínculo con ninguna de las distintas sociedades reformistas. Ahí encontramos a 20 miembros del Consejo que están vinculados únicamente con la correspondencia de Haller y otros 49 miembros del Consejo que pertenecen solamente a la Gran Sociedad, así como a 7 actores que pertenecen solo a estas tres y no a ninguna de las sociedades reformistas.

\footnotetext{
53 Tscharner 1909; Brunner 2009.

54 Im Hof y Capitani 1983.

55 Knill 1993.
} 
En cambio, aquellos actores que están conectados tanto con la élite política y social como con las sociedades reformistas se encuentran en el centro del dibujo. Los hermanos Vinzenz Bernhard Tscharner y Niklaus Emanuel Tscharner tienen la cifra más elevada de afiliaciones (6 conexiones cada uno), seguidos de otros miembros activos de la Sociedad Económica, que tienen 5 cada uno ${ }^{56}$.

Un poco más a la derecha tenemos a actores que carecen de conexiones directas con los centros institucionales de la élite política y social de la República Bernesa. Entre ellos figuran Johann Georg Zimmermann y Georg Ludwig de Auenstein, que no pertenecen al patriciado bernés porque son originarios de Brugg. Ambos desempeñaron un papel clave en el movimiento reformista de Berna. Los símbolos verdirrojos describen una clase interesante de actores estructuralmente equivalentes: miembros de la Sociedad Económica que se carteaban con Albrecht von Haller, la mayoría de los cuales eran médicos, farmacéuticos y clérigos que se comunicaban con Haller y con la Sociedad Económica en calidad de asistentes botánicos. En esa misma región encontramos a una serie de residentes de Vaud que están conectados de la misma forma con el movimiento reformista bernés a través de Haller y la Sociedad Económica, como Loys de Chessaux, Seigneux de Correvon, Tissot o Madame Vicat-Curtat. En este último grupo se incluyen todos los lausaneses. Si nos fijamos en la dimensión espacial, la razón es evidente: las ubicaciones de Waadtland están principalmente conectadas con la red de correspondencia de la Sociedad Económica (fig. 5): Orbe (26 cartas), Morges (29), Cottens (41), Nyon (56), Yverdon (57), Vevey (114) y -en primer lugar- Lausana (190).

Asimismo, con esta visualización, podemos preguntarnos cuáles son las posiciones de los actores de los ejemplos mencionados inicialmente. Albrecht von Haller, como miembro del Gran Consejo, presidente de la Sociedad Económica y figura clave de una red de correspondencia a escala europea -que en nuestra descripción se limita a su parte bernesa-, está situado en el centro. En el centro se encuentra también Johann Rudolf Tschiffeli. No obstante, a diferencia de Haller, él no pertenece al poder político (Gran Consejo), sino al centro de la sociedad (Gran Sociedad), así como a las dos sociedades de reforma política: la Sociedad de los Ciudadanos y la Sociedad Helvética. Por otro lado, Johann Jakob Dick no está representado ni el centro político ni en el social, y tampoco pertenece a ninguna de las dos sociedades reformistas mencionadas (de ahí que aparezca en el margen superior derecho).

56 Karl Emanuel Bonstetten y Alexander von Wattenwyl, los dos presidentes de la Sociedad Económica, y los miembros activos Emanuel von Graffenried y Franz Jakob von Tavel. Christoph Steiger y Niklaus Steiger son dos actores que tienen más de cinco vínculos y están situados en el centro, pero únicamente realizaron actividades secundarias dentro de la Sociedad Económica. 


\section{Conclusiones}

En primer lugar, se pueden clasificar los ejemplos mencionados inicialmente dentro del contexto general. La pregunta básica (si hay casos ejemplares o excepciones) se puede diferenciar según las distintas dimensiones aquí expuestas: cronológica, espacial, social y funcional. Al igual que en el caso de la transferencia de zanahorias, el de las plantas del género Rubia también tuvo lugar en una época en la que las dos redes estaban en pleno apogeo. Su dimensión europea se hace patente sobre todo en el caso de la transferencia de las zanahorias, ya que abarca tres zonas lingüísticas (alemán, inglés y francés). Como nexo de unión importante con otros espacios están los miembros honoríficos, con ayuda de los cuales la Sociedad Económica se integró en redes a nivel internacional, como fue el caso de los dos ejemplos mencionados inicialmente. Desde un punto de vista social, una serie de actores provenían de un entorno cercano al Estado (magistrados, responsables de la Administración y vicarios), lo que está en línea con la característica básica de la Ilustración Económica. El aumento de transferencias de plantas útiles, así como del conocimiento relacionado de esa misma manera, es una de las actividades fundamentales de ese movimiento. Una condición previa para el intercambio entre distintos espacios era una concordancia de los nombres locales, regionales e internacionales de las plantas, que solo podían obtenerse a través de la botánica científica. La interfaz más evidente con la República de las Letras era Albrecht von Haller, que desempeñó un papel clave en el mundo de la botánica en esa época. No obstante, el movimiento reformista agroeconómico también estaba relacionado con la República de las Letras a través del vicario Dick y del comerciante Collinson, los cuales mantenían una intensa correspondencia escrita con botánicos académicos.

La reconstrucción de las cadenas de acción de la transferencia de plantas relaciona a actores de grupos socialmente muy distintos. Esto se hace especialmente patente, entre otras cosas, a nivel local: Haller era un miembro de la magistratura que participó en la transferencia de las plantas del género Rubia, Tschiffeli era miembro del centro social y de los grupos de reforma política y Dick era un representante de los intelectuales sin ningún tipo de derechos políticos activos. El análisis de redes a varios niveles nos hace ser más conscientes de la cercanía espacial, social y funcional, así como de la distancia entre los actores.

Una de las objeciones más habituales relativas a la utilidad de los estudios históricos de redes es su abstracción en cuanto al espacio geográfico y al tiempo, mientras que las acumulaciones estadísticas de datos históricos en forma de series 
temporales, diagramas y mapas proporcionan únicamente una visión de los sistemas, y hacen caso omiso de la perspectiva individual. Al combinar ambas perspectivas en nuestro estudio de un análisis de redes a varios niveles, hemos demostrado cómo superar dichas carencias. En lugar de interpretar las distintas dimensiones de las redes como explicaciones alternativas, es precisamente su combinación la que nos permite entender mejor cómo contribuyeron esas redes a la transferencia de conocimientos.

\section{Referencias}

Bensaude-Vincent, Bernadette \& Bernardi, Bernard (2003). Rousseau et les sciences. París 2003: Editions L'Harmattan.

Billing, Robert (1765), An account of the culture of carrots and their great use in feeding and fattening cattle, published by desire of the Society for the Encouragement of Art, Manufactures, and Commerce at London. Londres: J. Dodsley.

Billing, Robert (1767), «Nachricht von dem Anbaue der Möhren, Daucus Sativus» en Abhandlungen und Beobachtungen durch die Oekonomische Gesellschaft zu Bern gesammelt, 2, pp. 129-155.

Bödeker, Hans Erich (1987). «Lessings Briefwechsel» en Hans Erich Bödeker y Ulrich Hermann. Über den Prozess der Aufklärung in Deutschland im 18. Jahrhundert. Personen, Institutionen und Medien. Gotinga: Vandenhoeck y Ruprecht, pp. 113-138.

Boschung, Urs et al. (2002). Repertorium zu Albrecht von Hallers Korrespondenz 1724-1777, 2 vols. Basilea: Schwabe.

Bosse, Heinrich (1997). «Die gelehrte Republik» en Hans-Wolf Jäger. 'Öffentlichkeit' im 18. Jahrhundert. Gotinga: Vandenhoeck y Ruprecht, pp. 51-76.

Bots, Hans y Wacquet, Françoise (1997). La République des Lettres. Berlín: De Boeck.

Brockliss, Laurence W.B (2002), Calvet's Web. Enlightenment and the Republic of Letters in Eighteenth-Century France. Oxford: University Press.

Brunner, Franz C. et al. (2009), Hôtel de Musique und Grande Société in Bern 1759-2009. Murten: Licorne. 
Dambourney, Louis-Alexandre (1763). «Mémoire sur la culture de la garance». Délibérations et mémoires de la Société Royale de l'agriculture de la généralité de Rouen, 1, pp. 241-274.

Dambourney, Louis-Alexandre (1767), «Extrait du mémoire sur la culture de la garance, présenté à la Société de Berne, en Février 1765, par Monsieur Tschiffely». Délibération et mémoires de la généralité de Rouen, pp. 269-301.

Dauser, Regina et al. (2008), Wissen im Netz. Botanik und Pflanzentransfer in europäischen Korrespondenznetzen des 18. Jahrhunderts. Berlín: Akademie Verlag.

Flückiger, Daniel y Stuber, Martin (2009), «Vom System zum Akteur. Personenorientierte Datenbanken für Archiv und Forschung» en André Kirchhofer et al., Nachhaltige Geschichte. Festschrift für Christian Pfister. Zúrich: Chronos, pp. 253-269.

Gerwer, Johann Jakob (1768), «Versuch in Anbauung der gelben Möhren». Abhandlungen und Beobachtungen durch die Oekonomische Gesellschaft zu Bern gesammelt, 1, pp. 70-75.

Grossetti, Michel; Barthe, Jean-François; y Chauvac, Nathalie (2011), «Studying Relational Chains from Narrative Material». Bulletin de Méthodologie Sociologique 110, pp. 11-25.

Hächler, Stefan (2008), «Avec une grosse boete de plantes vertes Pflanzentransfer in der Korrespondenz Albrecht von Hallers (1708-1777)» en Regina Dauser et al. (2008), Wissen im Netz. Botanik und Pflanzentransfer in europäischen Korrespondenznetzen des 18. Jahrhunderts. Berlín: Akademie Verlag, pp. 201-218.

Holenstein, André; Stuber, Martin; y Gerber-Visser, Gerrendina (2007), Nützliche Wissenschaft und Ökonomie im Ancien Régime. Akteure, Themen, Kommunikationsformen. (Cardanus Jahrbuch für Wissenschaftsgeschichte, 7). Heidelberg: Palatina.

Im Hof, Ulrich; y Capitani, François (1983), Die Helvetische Gesellschaft. Spätaufklärung und Vorrevolution in der Schweiz. 2 vols. Frauenfeld: Huber.

Jansen, Dorothea (2006), Einführung in die Netwerkanalyse. Wiesbaden: UTB (3. überarb. Aufl.). 
Knill, Ivo (1993), Die Société des citoyens und die Oekonomische Gesellschaft als Trägerinnen der Aufklärung in Bern. Berna: Lizentiatsarbeit Institut für Germanistik Univ. Bern.

Krempel, Lothar (2005), Visualisierung komplexer Strukturen. Grundlagen der Darstellung mehrdimensionaler Netzwerke. Fránkfurt y Nueva York: Campus.

Lowood, Henry E. (1991), Patriotism, Profit and the Promotion of Science in the German Enlightenment. The Economic Scientific Societies 1760-1815. Nueva York y Londres: Univ. of California.

Lüsebrink, Hans-Jürgen (2001), «Kulturtransfer - methodisches Modell und Anwendungsperspektiven» en Ingeborg Tömmel, Europäische Integration als Prozess von Angleichung und Differenzierung. Opladen: Leske \& Budrich, pp. 213-226.

Monti, Maria Teresa (1983-1994), Catalogo del Fondo Haller della Biblioteca Nazionale Braidense di Milano, 13 voll. Milán: Franco Angeli.

Popplow, Marcus (2010), Landschaften agrarisch-ökonomischen Wissens. Strategien innovativer Ressourcennutzung in Zeitschriften und Sozietäten des 18. Jahrhunderts. Münster et al.: Waxmann.

Reichardt, Rolf (2003), «Arbeitsperspektiven zur interkulturellen Kommunikation zwischen Ancien Régime und Moderne» en Thomas Fuchs y Sven Trakulhun, Das Eine Europa und die Vielfalt der Kulturen. Kulturtransfer in Europa 15001850. Berlín: Berliner Wissenschaftsverlag, pp. 27-46.

Roche, Daniel (1971), «Les primitifs du Rousseauisme: une analyse sociologique et quantitative de la correspondance de J.J. Rousseau». Annales 26, pp. 151-172.

Salzmann, Daniel (2009), Dynamik und Krise des ökonomischen Patriotismus. Das Tätigkeitsprofil der Oekonomischen Gesellschaft Bern 1759-1797. Nordhausen: Traugott Bautz.

Schneider, Ulrich Johannes (2005), Kultur und Kommunikation. Die europäische Gelehrtenrepublik im Zeitalter von Leibniz und Lessing. Wolfenbüttel: Harrassowitz.

Sigrist, René; y Widmer, Eric D. (2011), «Training links and transmission of knowledge in 18th Century botany: a social network analysis». REDES Revista hispana para el análisis de redes sociales, 21, 7, pp. 347-387.

Sigrist, René (en la versión impresa), «On the Social Characteristics of the 18th Century Botanists» en André Holenstein, Hubert Steinke y Martin Stuber (en la 
versión impresa), Scholars in Action. The Practice of Knowledge and the Figure of the Savant in the 18th Century. Leiden et al.: Brill.

Stapelbroek, Koen; y Marjanen, Jani (2012), The Rise of Economic Societies in the Eighteenth Century: Patriotic Reform in Europe and North America. Gordonsville: Palgrave Macmillan.

Steinke, Hubert (2000), Der nützliche Brief. Die Korrespondenz zwischen Albrecht von Haller und Christoph Jakob Trew 1733-1763, Basilea: Schwabe.

Steinke, Hubert (2003): «Archive database as advanced research tools: the HallerProject» en Maria Teresa Monti, Antonio Vallisneri. L'edizione del Testo scientifico d'età moderna. Florencia: L.S. Olschki, pp. 191-204.

Steinke, Hubert et al. (2008), Albrecht von Haller - Leben, Werk, Epoche. Gotinga: Wallstein.

Stuber, Martin; Hächler, Stefan; y Steinke, Hubert (2005), «Albrecht von Hallers Korrespondenz. Eine Gesamtanalyse» en Martin Stuber, Stefan Hächler y Luc Lienhard, Hallers Netz. Ein europäischer Gelehrtenbriefwechsel zur Zeit der Aufklärung. Basilea: Schwabe, pp. 3-216.

Stuber, Martin; y Lienhard, Luc (2007), «Nützliche Pflanzen. Systematische Verzeichnisse von Wild- und Kulturpflanzen im Umfeld der Oekonomischen Gesellschaft Bern 1762-1782» en André Holenstein et al., Nützliche Wissenschaft und Ökonomie im Ancien Régime. Akteure, Themen, Kommunikationsformen. (Cardanus Jahrbuch für Wissenschaftsgeschichte, 7). Heidelberg: Palatina, pp. 65-106.

Stuber, Martin (2005), «Brief und Mobilität bei Albrecht von Haller. Zur Geographie einer europäischen Gelehrtenkorrespondenz» en Johannes Burkhardt y Christine Werkstetter, Kommunikation und Medien in der Frühen Neuzeit (Historische Zeitschrift, Beiheft 41). Múnich: Oldenbourg, pp. 313-334.

Stuber, Martin; Hächler, Stefan; Krempel, Lothar; y Ruisinger, Marion Maria (2008), «Exploration von Netzwerken durch Visualisierung. Die Korrespondenznetze von Banks, Haller, Heister, Linné, Rousseau, Trew und der Oekonomischen Gesellschaft Bern» en Regina Dauser et al. (2008), Wissen im Netz. Botanik und Pflanzentransfer in europäischen Korrespondenznetzen des 18. Jahrhunderts. Berlín: Akademie Verlag, pp. 347-374.

Stuber, Martin (2008a), «Kulturpflanzentransfer im Netz der Oekonomischen Gesellschaft Bern» en Regina Dauser et al., Wissen im Netz. Botanik und 
Pflanzentransfer in europäischen Korrespondenznetzen des 18. Jahrhunderts. Berlín: Akademie Verlag, pp. 229-269.

Stuber, Martin (2008b), «Das Korrespondenznetz der Oekonomischen Gesellschaft Bern, 1759-1800» en Ulrich Johannes Schneider, Kulturen des Wissens im 18. Jahrhundert. Berlín y Nueva York: Walter de Gruyer, pp. 123-132.

Stuber, Martin et al. (2009), Kartoffeln, Klee und kluge Köpfe. Die Oekonomische und Gemeinnützige Gesellschaft des Kantons Bern OGG (1759-2009). Berna et al.: Haupt.

Stuber, Martin (2012), «Die Oekonomische Gesellschaft Bern als Kontaktzone im europäischen Austausch agrarisch-ökonomischen Wissens» en Regina Dauser y Lothar Schilling, Grenzen und Kontaktzonen. Rekonfigurationen von Wissensräumen zwischen Frankreich und den deutschen Ländern 1700-1850. discussions 7 (www.perspectivia.net).

Stuber, Martin (en la versión impresa), «Le réseau de correspondance d'Albert de Haller. Formation, perception, réception» en Jean Boutier y Emmanuelle Chapron, Conserver, archiver, éditer. Usages de la correspondance savante, XVIIe-XVIIIe siècles.

Tscharner, Louis de (1909), La Grande Société de Berne 1759-1909. Notice commémorative à l'occasion du cent cinquantième anniersaire de sa fondation. Berna: Stämpfli.

Tschiffeli, Johann Rudolf (1765), «Abhandlung vom Anbau der Färber-Röthe, oder des Grapps». Abhandlungen und Beobachtungen durch die Oekonomische Gesellschaft zu Bern gesammelt, 2, pp. 135-162.

Zaunstöck, Holger (1999), Sozietätslandschaft und Mitgliedstrukturen. Die mitteldeutschen Aufklärungsgesellschaften im 18. Jahrhundert. Tubinga: Max Niemeyer.

Zaunstöck, Holger y Meumann, Markus (2003), Sozietäten, Netzwerke, Kommunikation. Neue Forschungen zur Vergesellschaftung im Jahrhundert der Aufklärung. Tubinga: Max Niemeyer. 